\title{
Field-Induced Tomonaga-Luttinger Liquid Phase of a Two-Leg Spin-1/2 Ladder with Strong Leg Interactions
}

\author{
Tao Hong, ${ }^{1, *}$ Y. H. Kim,${ }^{2}$ C. Hotta, ${ }^{3}$ Y. Takano, ${ }^{2}$ G. Tremelling, ${ }^{4}$ M. M. Turnbull,${ }^{4}$ C. P. Landee,${ }^{4}$ H.-J. Kang, ${ }^{5}$ \\ N. B. Christensen, ${ }^{6}$ K. Lefmann, ${ }^{7}$ K. P. Schmidt, ${ }^{8}$ G. S. Uhrig, ${ }^{8}$ and C. Broholm ${ }^{5,9}$ \\ ${ }^{1}$ Neutron Scattering Science Division, Oak Ridge National Laboratory, Oak Ridge, Tennessee 37831, USA \\ ${ }^{2}$ Department of Physics, University of Florida, Gainesville, Florida 32611, USA \\ ${ }^{3}$ Department of Physics, Faculty of Science, Kyoto Sangyo University, Kyoto 603-8555, Japan \\ ${ }^{4}$ Carlson School of Chemistry and Department of Physics, Clark University, Worcester, Massachusetts 01610, USA \\ ${ }^{5}$ National Institute of Standards and Technology, Gaithersburg, Maryland 20899, USA \\ ${ }^{6}$ Laboratory for Neutron Scattering, ETH Zurich Paul Scherrer Institute, CH-5232 Villigen PSI, Switzerland \\ ${ }^{7}$ Nanoscience Center, Niels Bohr Institute, University of Copenhagen, DK-2100 Copenhagen, Denmark \\ ${ }^{8}$ Lehrstuhl für theoretische Physik I, TU Dortmund, Otto-Hahn-Straße 4, D-44221 Dortmund, Germany \\ ${ }^{9}$ Department of Physics and Astronomy, The Johns Hopkins University, Baltimore, Maryland 21218, USA \\ (Received 29 April 2010; revised manuscript received 19 July 2010; published 23 September 2010) \\ We study the magnetic-field-induced quantum phase transition from a gapped quantum phase that \\ has no magnetic long-range order into a gapless phase in the spin-1/2 ladder compound \\ bis(2,3-dimethylpyridinium) tetrabromocuprate (DIMPY). At temperatures below about $1 \mathrm{~K}$, the specific \\ heat in the gapless phase attains an asymptotic linear temperature dependence, characteristic of a \\ Tomonaga-Luttinger liquid. Inelastic neutron scattering and the specific heat measurements in both \\ phases are in good agreement with theoretical calculations, demonstrating that DIMPY is the first model \\ material for an $S=1 / 2$ two-leg spin ladder in the strong-leg regime.
}

DOI: 10.1103/PhysRevLett.105.137207

PACS numbers: 75.10.Jm, 75.40.Gb, 75.50.Ee

Gapped ground states comprising singlet pairs of spins are the prevalent nonmagnetic quantum disordered states in a variety of antiferromagnetic Heisenberg models [1-3]. Among those models, two-leg spin-1/2 ladders with antiferromagnetic rung and leg exchanges, $J_{\text {rung }}$ and $J_{\text {leg }}$, are the simplest whose ground states are yet nontrivial. These states give way to a Tomonaga-Luttinger liquid (TLL) — a critical state with fractional $S=1 / 2$ spinon excitations-at a magnetic-field-driven quantum critical point (QCP) [4].

Although the quantum phase transition at such a QCP has been extensively investigated theoretically [5-8], there have been few experimental studies because of the scarcity of real systems with the right energy scales. $\left(\mathrm{C}_{5} \mathrm{H}_{12} \mathrm{~N}\right)_{2} \mathrm{CuCl}_{4}$, which was originally thought to be a ladder material [9], later turned out to be a frustrated three-dimensional antiferromagnet [10]. In IPA- $\mathrm{CuCl}_{3}$ $[11,12]$, long-range magnetic order-also known as a Bose-Einstein condensation of magnons [1,13] — due to interladder interactions dominates the magnetic-field region above the QCP. Thus far, the only detailed report of a TLL in a two-leg spin-1/2 ladder has concerned $\left(\mathrm{C}_{5} \mathrm{H}_{12} \mathrm{~N}\right)_{2} \mathrm{CuBr}_{4}$, a strong-rung material with $J_{\text {leg }} / J_{\text {rung }} \approx$ $0.25[14,15]$. For deeper understanding of ladders, development of new materials with a wide range of $J_{\text {leg }} / J_{\text {rung }}$ will be crucial. Of special interest are materials in the strong-leg regime, $J_{\text {leg }} / J_{\text {rung }}>1$, since quantum fluctuations are more prominent in this regime and as a result the singlets will be less localized, a state reminiscent of the resonating valence bond liquid $[16,17]$.
In this Letter, we investigate a magnetic-field-induced quantum phase transition in $\left(\mathrm{C}_{7} \mathrm{H}_{10} \mathrm{~N}\right)_{2} \mathrm{CuBr}_{4}$, DIMPY for short, a new material in which the $\mathrm{CuBr}_{4}^{-2}$ radicals form two-leg spin ladders along the crystallographic $\boldsymbol{a}$ axis [18]. Our inelastic neutron scattering (INS) demonstrates that this compound is a spin-gapped quantum magnet with excellent one-dimensionality. Our specific-heat measurements reveal the presence of a TLL phase above the critical field $H_{c}=3.0(3) \mathrm{T}$, with no long-range order at least down to $150 \mathrm{mK}$. With the aid of perturbative continuous unitary transformations (PCUTs) and state-of-the-art density-matrix renormalization-group (DMRG) calculations, we determine the strengths of the rung and leg exchanges from the INS results in the gapped phase and the specific-heat results in the TLL phase with remarkable consistency, confirming that DIMPY is an ideal $S=1 / 2$ spin-ladder system in the strong-leg regime.

Single crystals of deuterated DIMPY were grown according to the method described in Ref. [18]. Promptgamma neutron activation analysis measurements showed that $67 \%$ of hydrogen sites are occupied by deuterium. The zero-field INS experiment was performed on SPINS at NIST with a single crystal of a $3.5 \mathrm{~g}$ mass and a $0.5^{\circ}$ mosaic spread. The measurements were made in the $(h, k, 0)$ and $(h, 0, l)$ reciprocal-lattice planes with a standard helium cryostat. The high-field INS experiment was performed on RITA II at SINQ, PSI. The sample consisted of two single crystals with a total mass of $2 \mathrm{~g}$ coaligned within $0.6^{\circ}$. The sample was oriented with the 
$(h, 0, l)$ plane horizontal and was cooled in a $15 \mathrm{~T}$ verticalfield cryomagnet. The data rate was increased by employing a multiblade crystal analyzer and a position sensitive detector [19]. A $\mathrm{Be}$ (or $\mathrm{BeO}$ ) filter was placed after the sample to remove high-order contamination, selecting a final neutron energy of 5.0 (or 3.7) $\mathrm{meV}$. The specific heat measurements were made with relaxation calorimetry at the NHMFL, Tallahasse, on a single crystal of an $8.2 \mathrm{mg}$ mass in fields up to $18 \mathrm{~T}$ applied parallel to the $\boldsymbol{c}$ axis.

Figure 1 summarizes the zero-field dispersion measured at $T=1.5 \mathrm{~K}$ by INS along three high symmetry directions in the reciprocal space [20]. We performed a global fit of all collected data to a dynamic spin correlation function with the approximate spin-gap dispersion $\epsilon(\boldsymbol{q})=$ $\sqrt{\Delta^{2}+v^{2} \sin ^{2}[2 \pi(0.5-h)]}$ [21], convolved with instrumental resolution, finding $\Delta=0.32(2) \mathrm{meV}, \quad v=$ 2.36(4) $\mathrm{meV}$. The individual data points shown in the figure were obtained by fitting a resolution-corrected line shape to each constant- $\boldsymbol{q}$ (or constant-energy) scan. Note that Figs. 1(a) and 1(b) are shown on a much finer scale than Fig. 1(c). Within a scale as small as $20 \mu \mathrm{eV}$, dispersion is absent along the $\boldsymbol{c}$ direction, and only a very weak dispersion, if any, of at most $50 \mu \mathrm{eV}$ is found along the $\boldsymbol{b}$ direction [22], indicating that DIMPY is an excellent one-dimensional (1D) system.

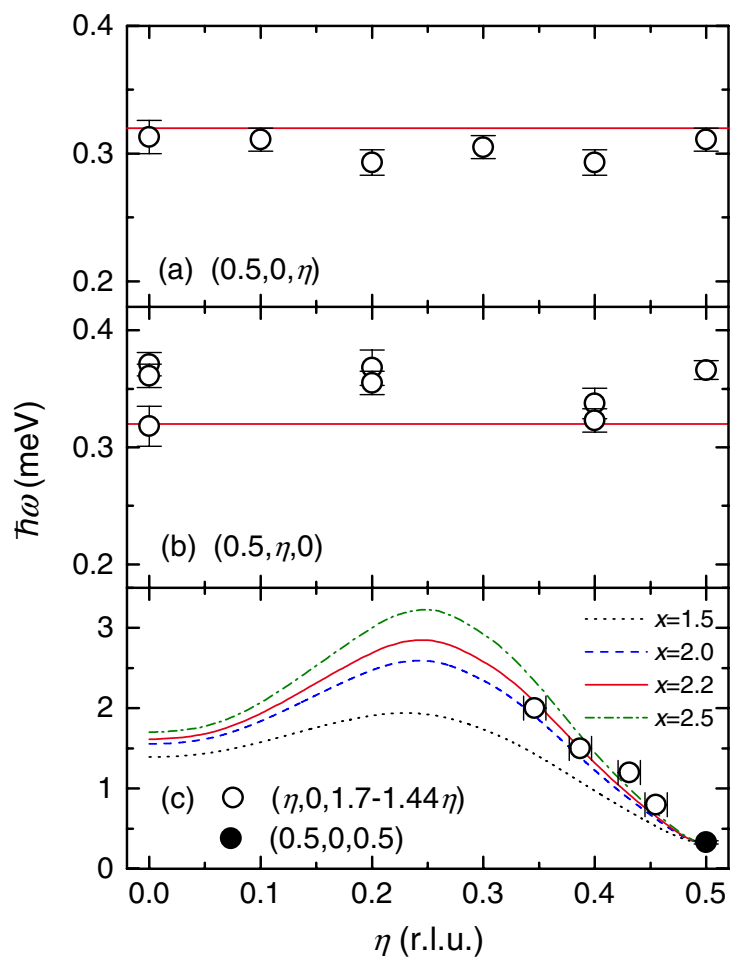

FIG. 1 (color online). Dispersion measured by INS in DIMPY at $T=1.5 \mathrm{~K}$ as a function of $h, k$, and $l$. Lines in (a) and (b) indicate the gap energy. Lines in (c) are from PCUT calculations for an AFH two-leg spin ladder for different values of $x=J_{\text {leg }} / J_{\text {rung }}$.
We have calculated the dispersion of an $S=1 / 2$ antiferromagnetic Heisenberg (AFH) spin-ladder system, using PCUTs [23] around the limit of isolated rungs. The series in $x=J_{\text {leg }} / J_{\text {rung }}$ is obtained in the thermodynamic limit [24] and is extrapolated in terms of an internal parameter [25] using Padé resummation, yielding reliable results for large $x$ especially for $\boldsymbol{q}$ close to the magnetic zone center. The lines in Fig. 1(c) are the dispersion for different values of $x$, calculated in conjunction with the accurate gap value $\Delta=0.32(2) \mathrm{meV}$. Best agreement with the data is obtained for $x=2.2(2)$, indicating that DIMPY is in the strong-leg regime.

Figure 2(a) shows the background-subtracted constant- $\boldsymbol{q}$ scan at the magnetic zone center $(0.5,0,0.9)$ at $T=1.5 \mathrm{~K}$ in different fields. The background was determined at zero field by making energy scans at $\boldsymbol{q}=(0.35,0,0.9)$ and $(0.65,0,0.9)$, away from the magnetic zone center, with the same instrument configuration and by fitting the results to a Gaussian profile over the range where no magnetic excitation is present. At zero field, the resolution-limited

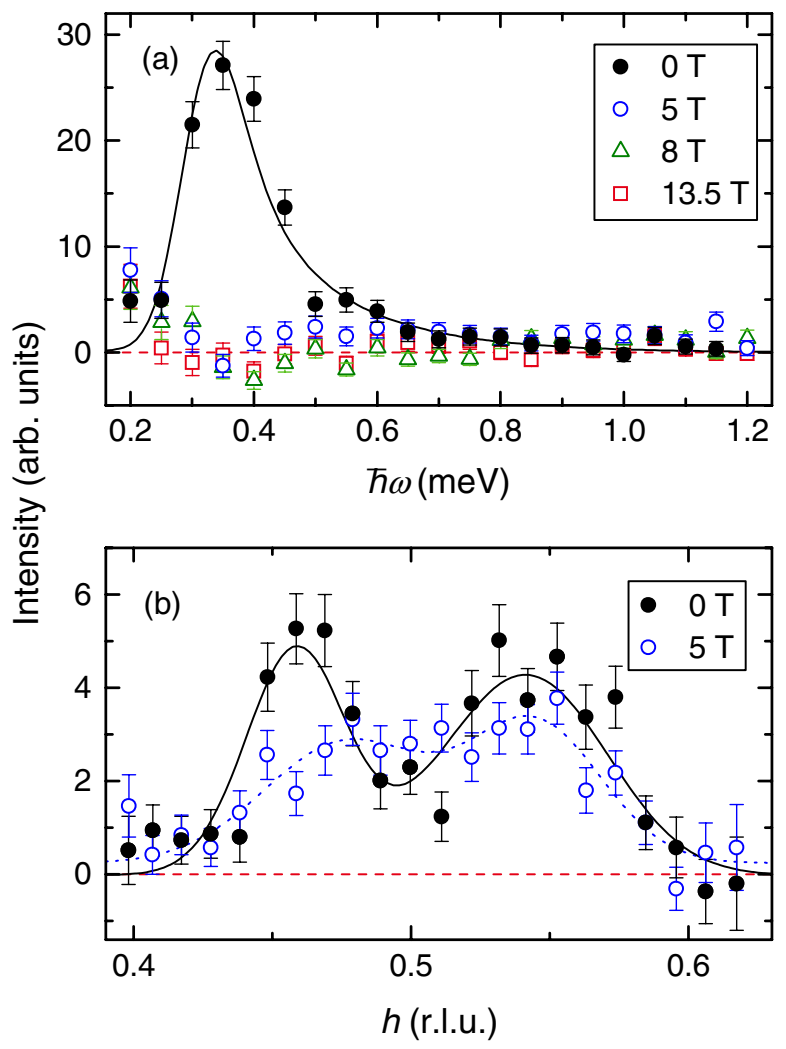

FIG. 2 (color online). (a) Background-subtracted constant- $\boldsymbol{q}$ scan in DIMPY at the magnetic zone center $(0.5,0,0.9)$ at $T=$ $1.5 \mathrm{~K}$ for magnetic fields $H=0,5,8$, and $13.5 \mathrm{~T}$. (b) Background-subtracted constant $\hbar \omega=0.7 \mathrm{meV}$ scans along the $(h, 0,1.7-1.44 h)$ direction at $T=1.5 \mathrm{~K}$ and $H=0$ and $5 \mathrm{~T}$. The dotted line is a guide for the eye. In both frames, solid lines are fits to a dynamic spin correlation function with the approximate spin-gap dispersion relation [21], convolved with instrumental resolution, and dashed lines indicate zero. 
peak indicates the location of the spin gap. Such a peak is absent at and above $5 \mathrm{~T}$, indicating that the magnetic field drives the system into a gapless critical phase.

To examine the magnetic excitation spectra at zero field and in the gapless phase, constant-energy scans were performed at $T=1.5 \mathrm{~K}$ for $\hbar \omega=0.7 \mathrm{meV}$ as shown in Fig. 2(b), where a constant background term has been subtracted. These measurements were made along the $(h, 0,1.7-1.44 h)$ direction to maximize the structure factor. The $\boldsymbol{q}$ resolution-limited peaks at zero field are from one-particle excitations. The low-energy feature in the gapless phase, at $5 \mathrm{~T}$, is clearly much broader than the experimental resolution, suggesting that it arises from a two-spinon continuum, not from one-particle excitations.

To augment the INS results, we measured the specific heat at $T<2.5 \mathrm{~K}$, as shown in Fig. 3. The phonon contribution was determined from the zero-field entropy $S=\int(C / T) d T$ and has been subtracted from the data at all fields. The nuclear-spin contribution has also been subtracted through a simultaneous fit to the data for all fields at temperatures below $700 \mathrm{mK}$.

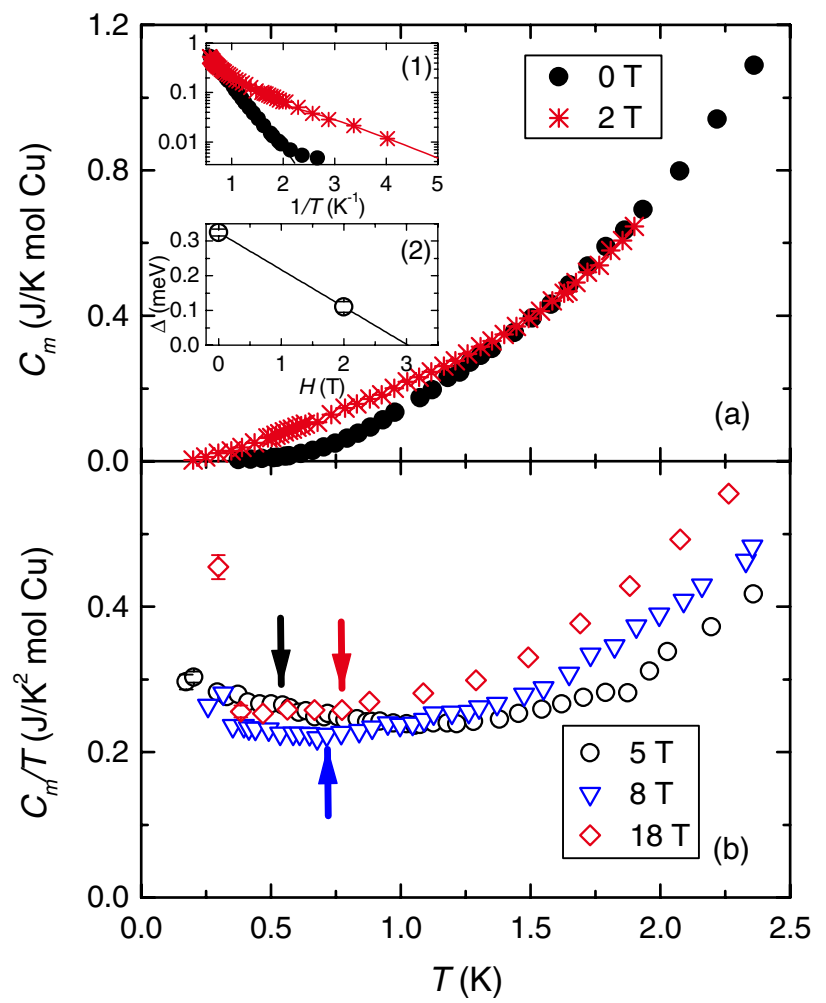

FIG. 3 (color online). Magnetic specific heat $C_{m}$ of DIMPY as a function of temperature $T$ for (a) $H<H_{c}$ and (b) $H>H_{c}$. In the latter region, the data have been plotted as $C_{m} / T$ after subtracting the nuclear-quadrupole contribution (see Ref. [27]). $v_{F}$ is extracted from data between $0.3 \mathrm{~K}$ and the upper limit of the $T$-linear region, indicated by an arrow. Inset (1) Semilog plot of the $H<H_{c}$ data against $1 / T$. Lines are fits to Eq. (1). Inset (2) Field dependence of the spin gap obtained from the data.
At zero field and $2 \mathrm{~T}$, exponentially activated behavior is found, as shown in the first inset to Fig. 3(a), providing additional clear evidence for a spin gap below a critical field. The specific heat of a gapped 1D AFH quantum magnet in the low-temperature limit is given by [26]:

$$
C(T)=\frac{\tilde{n} R}{2 \sqrt{2 \pi}}\left(\frac{\Delta}{k_{B} T}\right)^{3 / 2} \frac{\Delta}{v} e^{-\Delta / k_{B} T},
$$

where $\tilde{n}$ is the number of gapped low-energy modes and $R$ the gas constant. Fitted at $k_{B} T \ll \Delta$ to this expression, the zero-field data yield $\Delta=0.32(1) \mathrm{meV}$ - excellent agreement with the INS result-and $\tilde{n} / v=1.26(2)$ [27]. Taking $v=2.36(4) \mathrm{meV}$ from INS, we find $\tilde{n}=3.0(1)$, which unambiguously indicates the threefold degeneracy expected for a two-leg spin ladder. The field dependence of $\Delta$ is shown in the second inset to Fig. 3; a linear fit gives $H_{c}=3.0(3) \mathrm{T}$ in good agreement with $\Delta /\left(g \mu_{B}\right)=$ 2.8(2) $\mathrm{T}$, assuming $g=2.0$.

Above $H_{c}$, the specific heat shows remarkable behavior. There is no $\lambda$-like peak, indicative of a phase transition, at temperatures down to $150 \mathrm{mK}$ and magnetic fields up to $18 \mathrm{~T}$. Figure 3(b) shows the magnetic specific heat divided by temperature, $C_{m} / T$, at 5,8 , and $18 \mathrm{~T}$. As temperature decreases, $C_{m}$ reaches an asymptotic $T$-linear limit, characteristic of a TLL, before an upward deviation sets in-probably a precursor of long-range ordering due to weak interladder interactions [28]. The low-temperature specific heat of TLL is given by conformal field theory as $[29,30]$ :

$$
C(T)=\frac{\pi}{3} R \frac{k_{B} T}{v_{F}(H)},
$$

where $v_{F}$, the Fermi velocity, is the velocity of the gapless excitations. Using this equation, we extract $v_{F}=2.79(8)$, $3.27(11)$, and 2.89(9) $\mathrm{meV}$, respectively, from the specific heat at 5,8 , and $18 \mathrm{~T}$.

From these $v_{F}$ and $\Delta$, we now determine $x=J_{\text {leg }} / J_{\text {rung }}$ and $J_{\text {rung }}$. First, we perform DMRG calculations for $S=$ 1/2 AFH two-leg ladders [31], in conjunction with finitesize scaling, and obtain $v_{F} / J_{\text {leg }}$ as a function of $g \mu_{B} H / J_{\text {leg }}$ for fixed $x$ [32] and $\Delta / J_{\text {rung }}$ as a function of $x$ [33]. From this $\Delta / J_{\text {rung }}$ and $\Delta=0.32(2) \mathrm{meV}$ from the zero-field specific heat and INS, we find $J_{\text {leg }}$-which is $x J_{\text {rung }}$-for each $x$. With these $J_{\text {leg }}$, we then normalize the experimental values of $v_{F}$ and plot them with the theoretical results, as shown for $x=2$ and 2.5 in Fig. 4. Finally, comparison of experiment and theory in this plot yields $x=2.3(2)$, for which $\Delta / J_{\text {rung }}=0.409(6)$ and thus $J_{\text {rung }}=0.78(6) \mathrm{meV}$.

To summarize, DIMPY undergoes a quantum phase transition at $H_{c}=3.0(3) \mathrm{T}$ from a gapped phase to a Tomonaga-Luttinger liquid (TLL). Inelastic neutron scattering reveals the excellent one-dimensionality of this material and provides a firm value of the spin gap, $\Delta=0.32(2) \mathrm{meV}$, as does the specific heat. In the TLL 


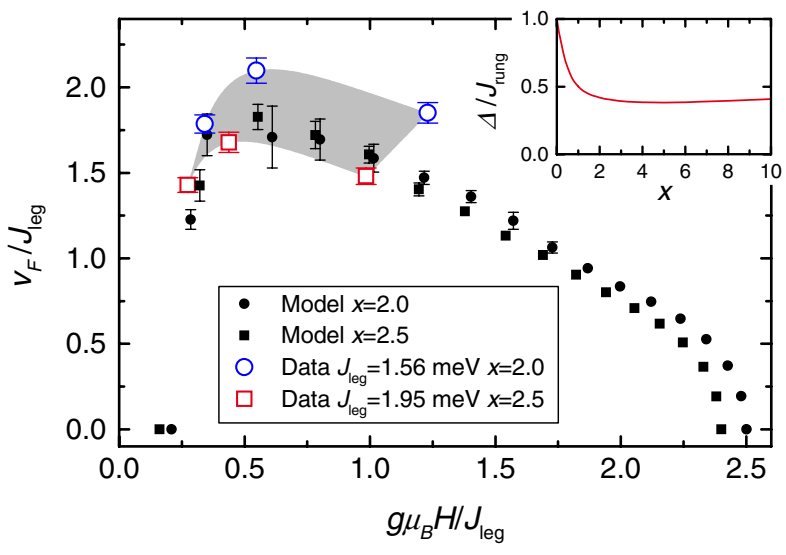

FIG. 4 (color online). Field dependence of the Fermi velocity $v_{F}$ scaled with $J_{\text {leg }}$. Filled symbols are DMRG results for $x=$ 2.0 and 2.5. Open symbols are from specific heat, scaled with $J_{\text {leg }}=1.56 \mathrm{meV} \quad(x=2.0)$ and $J_{\text {leg }}=1.95 \mathrm{meV} \quad(x=2.5)$, where $J_{\text {leg }}$ for each $x$ has been found from calculated $\Delta / J_{\text {rung }}$ (see the inset) and the spin gap $\Delta=0.32 \mathrm{meV}$. For $x$ from 2.0 to 2.5 , scaled experimental data lie in the shaded region.

phase, the specific heat attains characteristic $T$-linear behavior, yielding the Fermi velocity $v_{F}$ of the gapless excitations for the first time in any laboratory TLL. We obtain $J_{\text {rung }}=0.78(6) \mathrm{meV}$ from $\Delta$ and $v_{F}$, and the exchange ratio $x=2.2(2)$ from the zero-field dispersion and 2.3(2) from $\Delta$ and $v_{F}$. These are consistent with previous estimates, $J_{\text {rung }}=0.75 \mathrm{meV}$ and $x=1.94$, from magnetic susceptibility [18]. Three independent experiments yielding the exchange constants with consistency and in excellent agreement with theory establish DIMPY unambiguously as the first ideal realization of an $S=$ $1 / 2$ AFH two-leg ladder in the strong-leg regime, thus opening up an avenue for investigating the properties of such a ladder in this poorly explored regime.

We thank R. Paul for help with neutron activation analysis, and J.-H. Park and T.P. Murphy for help with cryogenics. The work at ORNL was partially funded by the Division of Scientific User Facilities, Office of BES, DOE. The work at JHU was supported by the NSF through Grants No. DMR-0306940 and No. DMR-0706553. C. H. was supported by Kakenhi Grants No. 19740218, No. 21110522, and No. 22014014 from the Ministry of Education, Science, Sports, and Culture of Japan. K.P.S. acknowledges ESF and EuroHorcs for funding through EURYI. The work at RITA II, PSI was supported by the Danish Natural Science Research Council under DANSCATT and by the Swiss NSF Contract No. PP002102831. The work at NIST utilized facilities supported in part by the NSF under Agreement No. DMR-0454672. The cryomagnet at PSI was partially funded by the Carlsberg Foundation. The NHMFL is supported by NSF Cooperative Agreement No. DMR-0654118, and by the State of Florida and the DOE. *hongt@ornl.gov

[1] T. Giamarchi, Ch. Rüegg, and O. Tchernyshyov, Nature Phys. 4, 198 (2008).

[2] T. Senthil et al., Science 303, 1490 (2004).

[3] T. Giamarchi, Quantum Physics in One Dimension (Oxford University Press, New York, 2004).

[4] F. D. M. Haldane, J. Phys. C 14, 2585 (1981).

[5] S. Sachdev, T. Senthil, and R. Shankar, Phys. Rev. B 50, 258 (1994).

[6] R. Chitra and T. Giamarchi, Phys. Rev. B 55, 5816 (1997).

[7] A. Furusaki and S.-C. Zhang, Phys. Rev. B 60, 1175 (1999).

[8] X. Wang and L. Yu, Phys. Rev. Lett. 84, 5399 (2000).

[9] G. Chaboussant et al., Phys. Rev. B 55, 3046 (1997).

[10] M. B. Stone et al., Phys. Rev. B 65, 064423 (2002).

[11] V. O. Garlea et al., Phys. Rev. Lett. 98, 167202 (2007).

[12] T. Hong, A. Zheludev, H. Manaka, and L.-P. Regnault, Phys. Rev. B 81, 060410(R) (2010).

[13] T. Giamarchi and A. M. Tsvelik, Phys. Rev. B 59, 11398 (1999).

[14] Ch. Rüegg et al., Phys. Rev. Lett. 101, 247202 (2008).

[15] B. Thielemann et al., Phys. Rev. Lett. 102, 107204 (2009).

[16] S. R. White, R. M. Noack, and D. J. Scalapino, Phys. Rev. Lett. 73, 886 (1994).

[17] Y. Xian, Phys. Rev. B 52, 12485 (1995).

[18] A. Shapiro et al., J. Am. Chem. Soc. 129, 952 (2007).

[19] C. R. H. Bahl et al., Nucl. Instrum. Methods Phys. Res., Sect. B 226, 667 (2004).

[20] The data shown in Fig. 1(c) are limited to wave vector transfers $0.35 a *$ and larger, because of the large incoherent scattering cross section and the decreasing spectral weight towards the magnetic zone boundary.

[21] T. Masuda et al., Phys. Rev. Lett. 96, 047210 (2006).

[22] The energy variation of the excitations in this direction may be an artifact due to slight sample misalignment and neutron self-shielding effects in the $(h, k, 0)$ plane.

[23] K. P. Schmidt and G.S. Uhrig, Mod. Phys. Lett. B 19, 1179 (2005).

[24] C. Knetter, K. P. Schmidt, and G. S. Uhrig, J. Phys. A 36, 7889 (2003).

[25] K. P. Schmidt, C. Knetter, and G. S. Uhrig, Acta Phys. Pol. B 34, 1481 (2003).

[26] M. Troyer, H. Tsunetsugu, and D. Würtz, Phys. Rev. B 50, 13515 (1994).

[27] The deviation of the zero-field data at $T \leq 0.5 \mathrm{~K}$ from Eq. (1), as seen in inset 1 to Fig. 3(a), is most likely a nuclear-quadrupole specific heat of bromine and copper. This term, $0.52(3) / \mathrm{T}^{2} \mathrm{~mJ} / \mathrm{Kmol}$, has been subtracted from the high-field data shown in Fig. 3(b).

[28] M. Hagiwara et al., Phys. Rev. Lett. 96, 147203 (2006).

[29] I. Affleck, Phys. Rev. Lett. 56, 746 (1986).

[30] H. W. J. Blöte, J. L. Cardy, and M. P. Nightingale, Phys. Rev. Lett. 56, 742 (1986).

[31] Our system size is up to $2 \times 80$ spins for the calculation of $v_{F} / J_{\text {leg }}$ and up to $2 \times 96$ spins for the calculation of $\Delta / J_{\text {rung }}$.

[32] Y. Maeda, C. Hotta, and M. Oshikawa, Phys. Rev. Lett. 99, 057205 (2007).

[33] $\left(\Delta / J_{\text {rung }}\right) / x$ gives $g \mu_{B} H / J_{\text {leg }}$ at $H_{c}$, above which the calculation of $v_{F} / J_{\text {leg }}$ is performed. 